\title{
Meta-optimization method for wavelet-based damage identification in composite structures
}

\author{
Andrzej Katunin, Piotr Przystałka \\ Silesian University of Technology, \\ Institute of Fundamentals of Machniery Design \\ ul. Konarskiego 18A, 44-100 Gliwice, Poland \\ Email: \{andrzej.katunin,piotr.przystalka\}@polsl.pl
}

\begin{abstract}
The damage identification problem is one of crucial problems during operation of machines' elements made of polymeric composites. Therefore the appropriate non-destructive techniques should be developed in order to detect and identify the damages with the best possible accuracy. Moreover, such methods should be applicable in various testing conditions. One of the intensively developed directions in non-destructive damage assessment is a class of methods based on wavelet analysis of modal shapes of vibration applied for a tested structure. The effectiveness of an algorithm is strongly dependent on the type of applied wavelet and its parameters. The proposed approach uses a combination of the wavelet-based damage identification algorithm with multi-objective meta-optimization in order to select optimal parameters of applied wavelets and determine a front of optimal non-dominated solutions. Based on these solutions the operator can choose the desired accuracy of damage identification with respect to the suitable computation time.
\end{abstract}

\section{INTRODUCTION}

$\mathbf{S}$ INCE the polymeric composites are more and more applicable as constructional materials in various industrial branches (e.g. automotive, aircraft and aerospace industries) and the manufactured elements are often subjected to critical loads during their operation, the development of appropriate damage identification methods, which will be able to detect and identify the damages specific for these materials, seems to be a necessity. From the majority of recently developed and applied non-destructive methods and techniques one could select a group of methods, which are based on analysis of vibration data of a tested structure. These methods have several advantages with respect to others, e.g. the possibility of carrying out on-field diagnostics, simplicity of measurements and concluding about the damage presence, a possibility of performing the measurements without unmounting the tested element from the machine, etc. However, for increasing the accuracy of detection and identification of damages the advanced signal processing methods are usually used.

One of the intensively developed approaches in the damage assessment problems is a wavelet-based analysis. Since the most of these problems are referred to the structural diagnostics of spatial domains the wavelet-based algorithms were extended for application on two-dimensional data. Numerous studies, both theoretical and experimental, were based on

The research project was financed by the National Science Centre (Poland) granted according the decision no. DEC-2011/03/N/ST8/06205. various wavelet transforms and various wavelets in order to obtain relevant information about damage state of a tested structure. A number of researchers developed their algorithms based on continuous wavelet transform (CWT) [1], [2], [3], [4] or stationary wavelet transform (SWT) [5]. The analysis of the applied wavelet-based algorithms was presented in [6]. The previous comparison studies of various wavelet transforms and various wavelets applied in the damage identification algorithm [7] show that the most accurate and computationally efficient algorithm is provided by application of the discrete wavelet transform (DWT) together with B-spline wavelets.

Following the recent advances in the field of improvement of wavelet-based damage identification methods one can notice that they are generally based on hybridization of the mentioned algorithm with various soft computing methods. There are numerous hybridizations of wavelet-based algorithm with artificial neural networks [8], [9], [10] as well as optimization algorithms: Krawczuk et al. [11] use genetic algorithms for improvement of cracks detection in beams, while the authors of [12] applied particle swarm optimization for improvement of detectibility of damages.

Further studies [14] of the first author allow developing a more efficient algorithm, which was based on fractional discrete wavelet transform (FrDWT) introduced in [15] with application of fractional B-spline wavelets. Based on the application of extended (two-dimensional) version of the fractional B-spline wavelets proposed in [16] it was possible to select the wavelet parameters suitable for the investigated problem in order to achieve the most accurate results of damage identification, which constitutes an improvement of accuracy with respect to integer-valued order of B-spline wavelets used previously [7], [17]. In order to select optimal parameters of the applied 2D fractional B-spline wavelets the authors hybridized a wavelet-based damage identification algorithm with various optimization algorithms (evolutionary algorithm, direct search algorithm, simulated annealing algorithm and particle swarm optimization) [18], which allowed for the further improvement of the damage identification effectiveness. The method was validated on numerical models and on experimental data achieved from vibrometric measurements of artificially damaged composite structures. Moreover, in the same paper, the authors surveyed the literature regarding the optimization problem of the wavelets' parameters mainly for 
the damage identification purposes. It was noted that, the optimization approaches might be included into two groups: classic methods and soft computing and heuristic ones. One of the main conclusions from this review was that there was the lack of sufficient methods to tune behavioural parameters of optimization algorithms in order to have a much more practical algorithm for damage identification, which could be implemented in the embedded system of the end-user device. In order to improve application abilities of the wavelet-based damage identification algorithm and evaluate the effectiveness of optimized wavelet parameters (and thus the effectiveness of damage detection and identification) with respect to the computational time the problem could be formulated as multiobjective meta-optimization one.

The meta-optimization is a quite novel approach, which found numerous applications in the engineering problems. One of the earliest attempts to meta-optimization can be found in [19], where the genetic algorithm was used in order to find best mutation and crossover rates for another lower-level genetic algorithm. In the next years, there were similar trials to this problem by many authors, e.g. see [20], [21], [22]. Also in this subject, the authors of the paper [23] discussed the most important issues related to tuning evolutionary algorithm parameters by means of various meta-optimization methods. Their main conclusion was that it was no matter what kind of tuner algorithms to be used in this task, because for each case, it was possible to get a much better result from evolutionary computations with meta-optimization than relying on own intuition and the usual parameter setting conventions. The similar strategy as in the case of evolutionary algorithms can be observed for other soft computing method. For example in [24], the authors proposed the concept in which a superordinate swarm ('superswarm') can be used to optimize the parameters of subordinate swarms ('subswarms'). Subordinate swarms were used for neural network training. Another point of the view is given in [25]. Branke and Elomari in their work proposed the method that could be used, in a single run, to identify the best parameter settings for all possible computational budgets. Their approach allows to save a lot of time. In the best of authors' knowledge the only application of meta-optimization in non-destructive testing with use of wavelet-based algorithm was presented in [26], where the authors performed electromagnetic measurements with appropriate post-processing in order to detect and identify cracks in walls of nuclear fission reactors.

In this study the authors developed an existing hybridized algorithm based on the results of the previous study [18]. The application of meta-optimization to the wavelet-based damage identification algorithm has several goals. In spite of the computational procedure implemented in [18] the authors determined common wavelets' parameters for all types of investigated damages. The parent optimization sub-algorithm in the meta-optimization algorithm was based on a double criterion problem, which allows to obtain a front of optimal non-dominated solutions dependent on the accuracy of damage identification vs. the computation time. Thus, one may decide which strategy should be applied, for instance, the worse solution with quick data processing or the best solution with long-time data processing.

\section{DAMAge IDENTIFICATION}

\section{A. Wavelet-based algorithm}

The algorithm of damage detection and identification was based on spatial FrDWT, which uses the two-dimensional Mallat's multi-resolution analysis, where B-spline scaling functions of fractional order $\beta_{\tau}^{\alpha}(x)$ constitute a space of the square-integrable subspaces $L^{2}\left(\mathbb{R}^{2}\right)$ and form a sequence of functional spaces $V_{i}$ in the form:

$$
\{0\} \subset \ldots \subset V_{-2} \subset V_{-1} \subset V_{0} \subset V_{1} \subset V_{2} \subset \ldots \subset L^{2}\left(\mathbb{R}^{2}\right) .
$$

The form of a scaling function of fractional order $\beta_{\tau}^{\alpha}(x)$ is defined by two parameters [15]: $\alpha \in \mathbb{R}$, which is an order of scaling function, and $\tau \in \mathbb{R}$, which is a shift parameter, and is as follows:

$$
\beta_{\tau}^{\alpha}(x)=\sum_{k=0}^{\infty}(-1)^{k}\left|\begin{array}{c}
\alpha+1 \\
k-\tau
\end{array}\right| \rho_{\tau}^{\alpha}(x-k),
$$

where

$$
\begin{gathered}
\rho_{\tau}^{\alpha}(x)=-\frac{\cos \pi \tau}{2 \Gamma(\alpha+1) \sin (\pi \alpha / 2)}|x|^{\alpha} \\
-\frac{\sin \pi \tau}{2 \Gamma(\alpha+1) \cos (\pi \alpha / 2)}|x|^{\alpha} \operatorname{sgn}(x),
\end{gathered}
$$

$\Gamma(\alpha+1)$ is the Euler $\gamma$-function, which allows for fractional factorization. The scaling $\beta_{\tau}^{\alpha}(x)$ and wavelet $\psi_{\tau}^{\alpha}(x)$ functions hold two-scale relations [15], [16]. For the cases when $\alpha \notin \mathbb{Z}$ and $\tau=(\alpha+1) / 2$ the integer-valued B-spline wavelets can be obtained.

Following the method of complexification of B-spline wavelets of fractional order based on generation of Hilbert transform pairs of them proposed in [16] it is possible to obtained direction-oriented 2D complex wavelets. The complexification is based on a combination of wavelets with the same order $\alpha$, but different shift parameters $\tau$ in the form:

$$
\psi_{\tau}^{\alpha}(x)=\psi_{\tau}^{\alpha}(x)+j \psi_{\tau+1 / 2}^{\alpha}(x), j^{2}=-1 .
$$

Considering that the analytic wavelet has a form [16]:

$$
\psi^{\alpha}(x)=\psi+j \mathcal{H}\{\psi\},
$$

where $\mathcal{H}$ denotes a Hilbert transform, the $2 \mathrm{D}$ complex wavelets have the following form [16]:

$$
\begin{array}{r}
\psi_{1}(\mathbf{X})=\psi(x) \phi(y)+j \mathcal{H} \psi(x) \phi(y), \\
\psi_{2}(\mathbf{X})=\psi(x) \mathcal{H} \phi(y)+j \mathcal{H} \psi(x) \mathcal{H} \phi(y), \\
\psi_{3}(\mathbf{X})=\phi(x) \psi(y)+j \phi(x) \mathcal{H} \psi(y), \\
\psi_{4}(\mathbf{X})=\mathcal{H} \phi(x) \psi(y)+j \mathcal{H} \phi(x) \mathcal{H} \psi(y), \\
\psi_{5}(\mathbf{X})=2^{-1 / 2}(\psi(x) \psi(y)-\mathcal{H} \psi(x) \mathcal{H} \psi(y)) \\
+2^{-1 / 2} j(\psi(x) \mathcal{H} \psi(y)+\mathcal{H} \psi(x) \psi(y)), \\
\psi_{6}(\mathbf{X})=2^{-1 / 2}(\psi(x) \psi(y)+\mathcal{H} \psi(x) \mathcal{H} \psi(y)) \\
+2^{-1 / 2} j(\psi(x) \mathcal{H} \psi(y)-\mathcal{H} \psi(x) \psi(y)),
\end{array}
$$


where $\mathbf{X}=(x, y)$ denotes $2 \mathrm{D}$ signal and $2^{-1 / 2}$ is used for scaling the wavelets. The wavelets (6) are oriented along the primal direcrions: $\theta_{1}=\theta_{2}=0, \theta_{3}=\theta_{4}=\pi / 2, \theta_{5}=\pi / 4$ and $\theta_{6}=3 \pi / 4$, which allows for increasing detectability of damages located in these directions. The decomposition graphical example can be found in [18].

Considering that discrete-type wavelet transforms could be expressed as a set of high-pass and low-pass filters, the decomposition procedure could be presented in the form of pairs of filters along the specific directions of a signal $\mathbf{X}$. Considering (4) and (6) one can obtain six complex subbands $\mathbf{w}^{P}, P=1, \ldots, 6$, after the decomposition (see [16], [18] for details).

In order to ensure sensitivity of an algorithm to all possible orientations of spatial damages the real parts of $\mathbf{w}^{P}$ are normalized according to the Euclidean metric. Moreover, considering the strong dependence between magnitudes of displacements of modal shapes and obtained coefficients after decomposition it is suitable to consider multiple modal shapes during the analysis. The resulted post-processing expression with respect to $M$ considered modes takes a form:

$$
\mathbf{W}=\sum_{M}\left|\sum_{P} \Re\left(\mathbf{w}_{M}^{P}\right)^{2}\right| .
$$

Based on the above-presented algorithm the damage identification procedure was performed. As was mentioned earlier, the wavelets were defined by $\alpha$ and $\tau$, thus these parameters were selected for the optimization procedure. The goal of optimization is to find suitable values for $\alpha$ and $\tau$, which allow for obtaining of best results in detection and localization of damages.

\section{B. Optimization procedure for searching values of $\alpha$ and $\tau$}

The main goal of the optimization procedure is to adjust the fractional order $\alpha$ and the shift factor $\tau$ in order to obtain the best properties of the damage identification algorithm. In the previous paper [18], the authors proved that the single optimization method could be successfully applied in this kind of tasks. Hence, the optimization problem can be written as follows:

$$
\begin{array}{ll}
\text { Minimize } & U(\alpha, \tau) \\
\text { subject to } & \alpha^{(L)} \leq \alpha \leq \alpha^{(U)}, \tau^{(L)} \leq \tau \leq \tau^{(U)}
\end{array}
$$

where $\alpha^{(L)}, \alpha^{(U)}, \tau^{(L)}, \tau^{(U)}$ are the lower and upper values of the boundary constraints that should be chosen taking into account the properties of the wavelet. The global criterion method [27] is used to create a single objective function $U(\alpha, \tau)$. In this way, an indirect utility function can be expressed in its simplest form as the weighted exponential sum:

$$
U(\alpha, \tau)=c_{1}\left[1+\sum_{i=1}^{T(p)} \max \left(\mathbf{W}_{i}\right)\right]^{-\lambda}+c_{2}\left[\sum_{i=1}^{n} \sum_{j=1}^{n} w_{i, j}^{*}\right]^{\lambda}
$$

where $c_{i}$ are weights indicating the relative significance of elements in the sum, the exponent $\lambda$ determines the extent to which a method is able to capture all of the Pareto-optimal points for either convex or non-convex criterion spaces, detail coefficients in the matrix $\mathbf{W}_{i}$ are computed using the recurrence relation proposed in [18], $w_{i, j}^{*}$ is an element of the matrix $\mathbf{W}_{T(p)}$. The function $T(p)$ can be defined using the following expression:

$$
T(p)=\operatorname{card}\left\{w_{i, j}: \forall i, j \in\{1,2, \ldots, n\} \quad w_{i, j} \geq p \max (\mathbf{W})\right\}
$$

where $n$ is the size of the matrix $\mathbf{W}$, whereas $p$ is the ratio between the greatest magnitudes and the other detail coefficients in the matrix $\mathbf{W}$. The value of this parameter should be chosen arbitrarily from the range $[0,1]$.

It is very important to have the physical interpretation of the formulated objectives. The first component of the weighted exponential sum (9) describes the values of detail coefficients that have the greatest magnitudes. This criterion can be interpreted as follows. If the damage occurred somewhere in the composite plate then the result of this would be locally affected on the values of displacements of modal shapes. The second component of the sum is correlated with the blurring of the regular form or forms which indicate the damage. It can be stated that the values of $w_{i, j}^{*}$ in the matrix represent the blurring of the regular form.

The authors showed in their previous paper [18] that the problem, which has been formulated in the form of (9) could be solved using heuristic optimization algorithms. As it was presented in their work, an evolutionary algorithm, a direct search algorithm, a simulated annealing algorithm and a particle swarm optimization algorithm could be adopted. The main problem in this kind of approaches is to find the compromise between the time computational complexity of an algorithm and the accuracy of a solution. These factors are strongly dependent on properties and values of the relevant parameters (behavioural parameters) of these algorithms.

\section{MetA-Optimization METHOD}

The idea of meta-optimization which is also known in the literature as super-optimization or hyper-heuristic is to apply one optimization technique to adjust another optimization technique. In this paper, the meta-optimization strategy corresponding to the data flow diagram presented in Fig. 1 is used in order to search the space of behavioural parameters. As it can be seen, the meta-optimization algorithm (MAC) evaluates a meta-objective function whereas the main optimization algorithm (OAE) computes the cost function in order to find an optimal solution with the minimum time complexity and maximum accuracy.

Meta-optimization concept can be realized in different ways, however one of the most promising approaches employs the multi-objective optimization algorithm. Consequently, the main purpose of the meta-optimization process is to tune values of behavioural parameters of the main optimization 


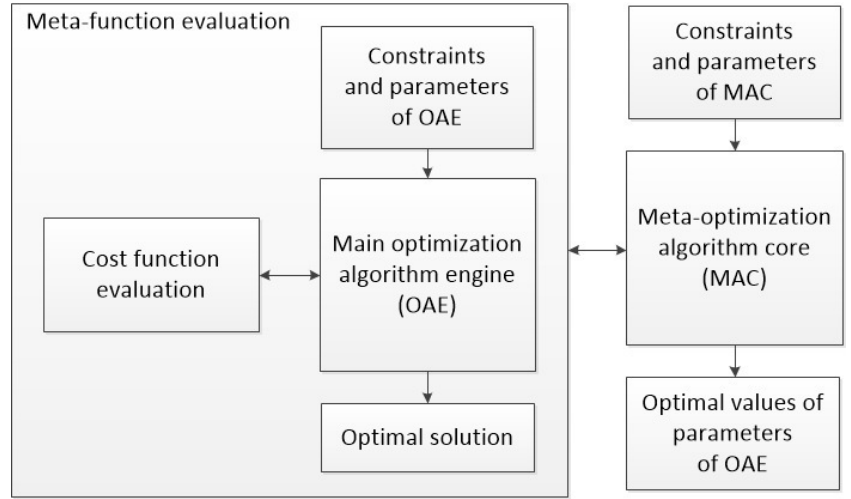

Fig. 1: A data flow diagram of the meta-optimization method

algorithm in order to minimize a multiple objective function U. This function can be formulated taking into account two fundamental criteria. The first criterion (MBF) corresponds to the estimation of the accuracy of a solution, whereas the second one (FES) represents the time computational complexity of the main optimization algorithm. When one assumes that both objectives are not conflicted then the multi-objective meta-optimization problem can be stated as follows:

$$
\begin{array}{ll}
\text { Minimize } & \mathbf{U}(\xi)=[\operatorname{MBF}(\xi) \\
\text { subject to } & \Omega(\xi)
\end{array}
$$

where $\xi$ is the set of properties of the main algorithm, $\Omega$ represents boundaries and constraints in the meta-optimization process. The accuracy of the solution that is found by the main optimization algorithm, can be computed as the mean value of best scores of the cost function evaluations. On the other hand, the time complexity is approximated using the total number of cost function evaluations in the same algorithm.

Generally, multi-objective optimization problems do not have single global solution, and therefore there is the need to investigate a set of points, each of which satisfies the objectives. Due to this, in the present study, predominant Pareto optimality concept is mainly used. A solution is Pareto optimal if there is no other solution that improves at least one objective function without detriment another function [27]. It is often viewed the same as a non-dominated solution.

It is reasonable to expect that each of multi-objective versions of soft computing methods indicated in the previous section to be applicable in the task of meta-optimization. Nevertheless, the authors propose to use a much less complicated algorithm in the main optimization engine, while a more advanced approach in the meta-optimization core. In such manner, it is possible to obtain general values of relevant parameters of the main algorithm that can easily be implemented in the embedded system of the end-user device.

\section{RESEARCH RESULTS}

The advantages and limitations of the proposed metaoptimization method were attempted in two separate experiments. The aim of the first case study was to validate the performance of the meta-optimization approach over a set of well-practised test functions. The second experiment dealt with the useful application of the elaborated method for waveletbased damage identification in composite structures. It was decided that, the engine of the main optimization algorithm was prepared using the particle swarm optimization algorithm (PSO-OAE), while the core of the meta-optimization process was implemented by means of the multi-objective evolutionary algorithm (MOEA-MAC). MOEAs are known in the literature as the heuristic methods for solving optimization problems, which are based on the natural selection process that mimics biological evolution. The MOEA recommended in [28] is utilized herein to solve the meta-optimization problem defined as (11). Well-known and often practised genetic operators for multi-objective optimization are applied to obtain the convergence of a solution. In such manner, the problem of finding values of behavioural parameters is solved by computing the Pareto front, hence the set of evenly distributed nondominated optimal solutions are determined. PSO is also classified into heuristic approaches, however this is a populationbased stochastic optimization technique, which is inspired by simulation of social behaviour. In this paper, PSO proposed by [29] is adopted and applied to search for the optimal values of $\alpha$ and $\tau$. The both optimization algorithms were implemented in the MATLAB ${ }^{\circledR}$ environment using Genetic Algorithm and Particle Swarm Optimization Toolboxes.

\section{A. Benchmark tests}

In the first step, the proposed method was verified using selected benchmark functions proposed in the CEC'2008 Special Session and Competition on Large Scale Global Optimization [30]. Due to the nature of the main problem formulated in this study, the authors decided to select the following test functions:

- $F_{3}$ : Shifted Rosenbrock's function

$$
F_{3}(\mathbf{x})=\sum_{i=1}^{D-1}\left(100\left(z_{i}^{2}-z_{i+1}\right)^{2}\right)
$$

where $\mathbf{z}=\mathbf{x}-\mathbf{o}+\mathbf{1}, \mathbf{x}=\left[x_{1}, x_{2}, \ldots, x_{D}\right], \mathbf{x} \in$ $[-100,100]^{D}, \mathbf{o}=\left[o_{1}, o_{2}, \ldots, o_{D}\right]$ is the shifted global optimum $\mathbf{x}^{*}=\mathbf{o}, F_{3}\left(\mathbf{x}^{*}\right)=0$.

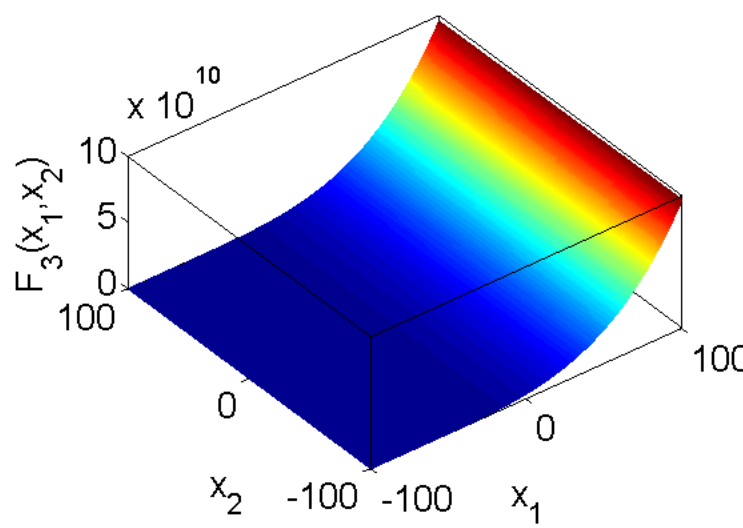


- $F_{4}$ : Shifted Rastrigin's function

$$
F_{4}(\mathbf{x})=\sum_{i=1}^{D}\left(z_{i}^{2}-10 \cos \left(2 \pi z_{i}\right)+10\right)
$$

where $\mathbf{x} \in[-5,5]^{D}, \mathbf{z}$ is the same as in the previous function, the shifted global optimum $\mathbf{x}^{*}=\mathbf{o}, F_{4}\left(\mathbf{x}^{*}\right)=$ 0 .

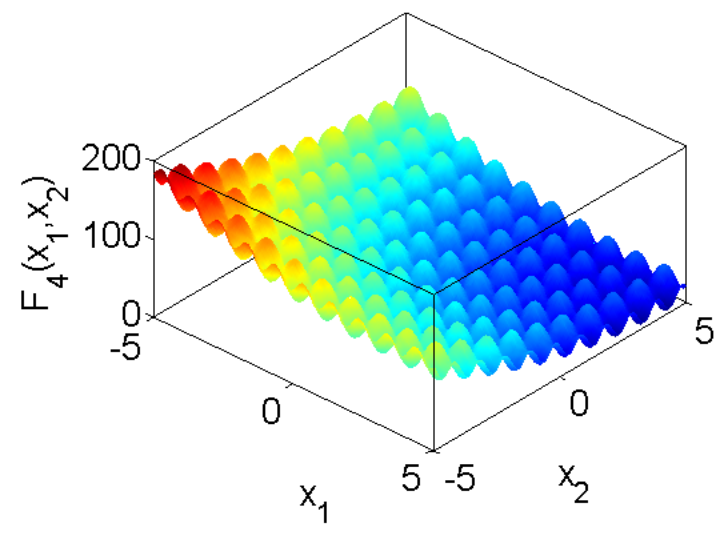

- $F_{5}$ : Shifted Griewank's function

$$
F_{5}(\mathbf{x})=\sum_{i=1}^{D} \frac{z_{i}^{2}}{4000}-\prod_{i=1}^{D} \cos \left(\frac{z_{i}}{\sqrt{i}}\right)+1
$$

where $\mathbf{z}=(\mathbf{x}-\mathbf{o}), \mathbf{x} \in[-600,600]^{D}$, the shifted global optimum $\mathbf{x}^{*}=\mathbf{o}, F_{4}\left(\mathbf{x}^{*}\right)=0$.

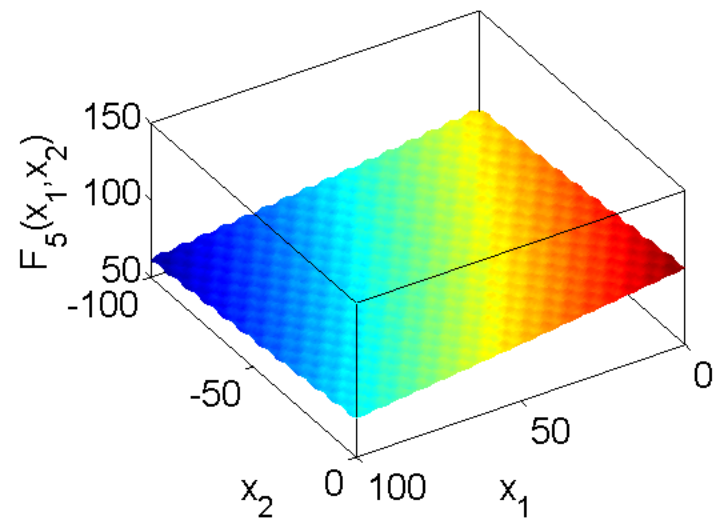

- $F_{6}$ : Shifted Ackley's function

$$
\begin{aligned}
& F_{6}(\mathbf{x})=-20 \exp \left(-0.2 \sqrt{\frac{1}{D} \sum_{i=1}^{D} z_{i}^{2}}\right)- \\
&-\exp \left(\frac{1}{D} \sum_{i=1}^{D} \cos \left(2 \pi z_{i}\right)\right)+20+e
\end{aligned}
$$

where $\mathbf{x} \in[-32,32]^{D}, \mathbf{z}$ is the same as in $F_{5}$ function, the shifted global optimum $\mathbf{x}^{*}=\mathbf{o}, F_{6}\left(\mathbf{x}^{*}\right)=0$.

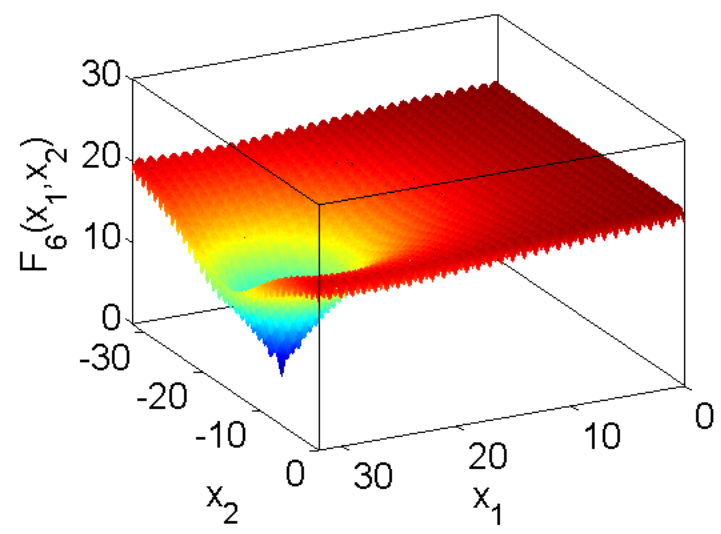

- $F_{7}$ : FastFractal 'DoubleDip' function

$$
F_{7}(\mathbf{x})=\sum_{i=1}^{D} \lambda_{1}\left(x_{i}+\lambda_{2}\left(x_{(i \bmod D)+1}\right)\right)+1720
$$

where

$$
\begin{aligned}
& \text { where } \\
& \lambda_{1}(x) \approx \sum_{k=1}^{3} \sum_{1}^{2^{k-1}} \sum_{1}^{\hat{o}_{2}} \lambda_{3}\left(x, \hat{o}_{1}, \frac{1}{2^{k-1}\left(2-\hat{o}_{1}\right)}\right), \\
& \lambda_{2}(y)=4\left(y^{4}-2 y^{3}+y^{2}\right), \\
& \lambda_{3}(x, c, s)=\left\{\begin{array}{l}
\left(-6144(x-c)^{6}+3088(x-c)^{4}\right. \\
\left.-392(x-c)^{2}+1\right) s, x \in(-0.5,0.5) \\
0, \text { otherwise }
\end{array},\right.
\end{aligned}
$$

and $\mathbf{x} \in[-1,1]^{D}$, the global optimum is unknown, $F_{7}\left(\mathbf{x}^{*}\right)$ is also unknown, $\hat{o}_{1}$ is a double precision variable, pseudo-randomly chosen, with seed $o_{1}$, with equal probability from the interval $[0,1], \hat{o}_{2}$ is an integer variable, pseudo-randomly chosen, with seed $o_{2}$, with equal probability from the set $\{0,1,2\}$.

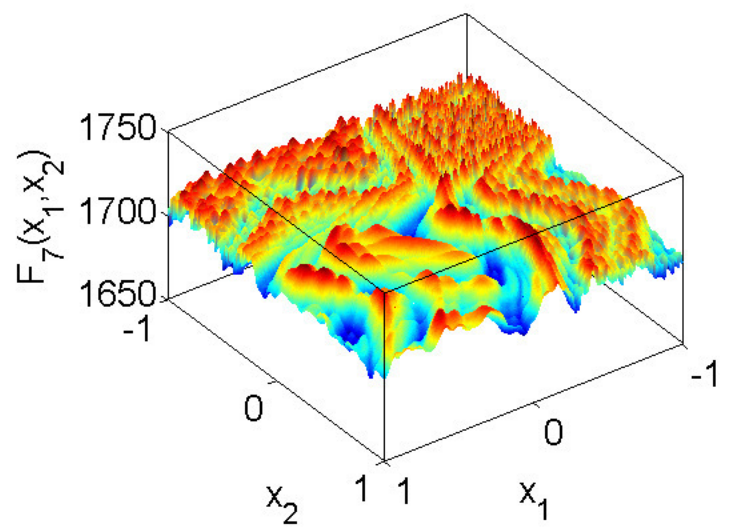

The task of the main optimization was defined as a continuous minimization problem. As it was mentioned above, the extreme was found with the help of the PSO-OAE. The cost function in the PSO-OAE was computed using one of the benchmark functions (12-16). In this algorithm only few parameters of the algorithm are relevant to guarantee, as far as possible, to find the optimal solution of the problem. Therefore, behavioural parameters such as the population size, the total number of generations, the social and cognitive 
attraction coefficients were taken into account during the metaoptimization process realized by means of MOEA-MAC. For each function, the lower and upper boundaries of the PSOOAE were assumed corresponding to functions' properties (12-16), whereas in the case of MOEA-MAC boundaries were declared in such a way, that the population size was equal from $D$ to $2 D$, the total number of generations from 5 to 100 , the cognitive and social attraction from 0 to 4 . The heuristic rules given in the literature were employed to get the best results from the MOEA. The fitness function was declared following to (11), where MBF was computed by averaging the best fitness function (for ten trials) and FES was obtained as the product of the population size and the total number of generations of the OAE. It was decided that individuals in the population of the MOEA were composed of genes representing real numeric values of behavioural parameters (the integer parts of the population size and generations parameters were used during the computations). The total number of generations of MOEA was set to 30 . The population size of this algorithm was equal 40 . The feasible population method was adapted to create a random well-dispersed initial population that satisfies all bounds in (11). Fitness scaling was realized using the rank method, whereas the selection of the parents to the next generation was achieved by applying the stochastic uniform method. Additionally, two reproduction options - the elite count and crossover fraction were chosen. The first one specifies the number of individuals that are guaranteed to survive to the next generation (it was equal 2 ). The second deals with the fraction of the next generation, other than elite children, that are produced by crossover. It was decided to use a heuristic crossover operator where the userdefined parameter was set to 1.2 , and the crossover probability was equal 0.8 . The remaining individuals are mutation children and they were obtained using the adaptive feasible method.

TABLE I: Optimal values of behavioural parameters for benchmark functions

\begin{tabular}{|c|c|c|c|c|c|}
\hline Function & $D$ & $\begin{array}{c}\text { Cognitive } \\
\text { attraction }\end{array}$ & $\begin{array}{c}\text { Social } \\
\text { attraction }\end{array}$ & Generations & $\begin{array}{c}\text { Population } \\
\text { size }\end{array}$ \\
\hline \multirow{2}{*}{$F_{3}$} & 2 & 2.180 & 0.706 & 74 & 4 \\
& 100 & 1.019 & 1.164 & 99 & 196 \\
\hline \multirow{2}{*}{$F_{4}$} & 2 & 0.789 & 2.267 & 69 & 4 \\
& 100 & 1.393 & 1.319 & 96 & 100 \\
\hline \multirow{2}{*}{$F_{5}$} & 2 & 0.712 & 1.125 & 88 & 4 \\
& 100 & 1.362 & 1.039 & 99 & 190 \\
\hline \multirow{2}{*}{$F_{6}$} & 2 & 0.350 & 0.826 & 100 & 4 \\
& 100 & 1.290 & 1.375 & 81 & 188 \\
\hline \multirow{2}{*}{$F_{7}$} & 2 & 0.988 & 0.920 & 67 & 4 \\
& 100 & 1.055 & 1.474 & 99 & 142 \\
\hline
\end{tabular}

The meta-optimization process was carried out for two cases $D=2$ and $D=100$. The achieved results are presented in Tab. I. Besides, in Figs. 2(a,c,e-k) there are given graphs with the visualisation of selected Pareto fronts (for functions $F_{3}, F_{6}$ and $F_{7}, D=2$ and $D=100$, respectively) based on which the optimal values of behavioural parameters were chosen. In this case study, the authors selected non-dominated optimal solutions that were characterized by the highest accuracy of the cost function (in a statistic sense) with the minimum as
TABLE II: Optimization results for different selection strategies of the behavioural parameter values

\begin{tabular}{|c|c|c|c|c|c|}
\hline Function & Case & MAX & MIN & AVG & STD \\
\hline$F_{3}$ & $\circ$ & $5.167 \mathrm{E}+01$ & $2.607 \mathrm{E}-01$ & $2.260 \mathrm{E}+01$ & $2.018 \mathrm{E}+01$ \\
$D=2$ & $\square$ & $1.470 \mathrm{E}+02$ & $5.549 \mathrm{E}-02$ & $6.384 \mathrm{E}+01$ & $5.887 \mathrm{E}+01$ \\
& $\triangle$ & $1.512 \mathrm{E}+02$ & $3.662 \mathrm{E}-01$ & $6.182 \mathrm{E}+01$ & $5.185 \mathrm{E}+01$ \\
\hline \multirow{2}{*}{$F_{3}$} & $\circ$ & $3.032 \mathrm{E}+09$ & $5.057 \mathrm{E}+08$ & $1.274 \mathrm{E}+09$ & $7.103 \mathrm{E}+08$ \\
$D=100$ & $\square$ & $5.000 \mathrm{E}+09$ & $1.382 \mathrm{E}+09$ & $2.675 \mathrm{E}+09$ & $1.345 \mathrm{E}+09$ \\
& $\triangle$ & $3.734 \mathrm{E}+09$ & $7.893 \mathrm{E}+08$ & $1.773 \mathrm{E}+09$ & $8.167 \mathrm{E}+08$ \\
\hline$F_{4}$ & $\circ$ & $8.955 \mathrm{E}+00$ & $1.644 \mathrm{E}-03$ & $2.451 \mathrm{E}+00$ & $3.071 \mathrm{E}+00$ \\
$D \stackrel{=}{=} 2$ & $\square$ & $2.487 \mathrm{E}+01$ & $1.079 \mathrm{E}-06$ & $6.369 \mathrm{E}+00$ & $7.404 \mathrm{E}+00$ \\
& $\triangle$ & $2.487 \mathrm{E}+01$ & $3.473 \mathrm{E}-07$ & $3.880 \mathrm{E}+00$ & $7.627 \mathrm{E}+00$ \\
\hline \multirow{2}{*}{$F_{4}$} & $\circ$ & $9.684 \mathrm{E}+02$ & $7.938 \mathrm{E}+02$ & $8.869 \mathrm{E}+02$ & $6.705 \mathrm{E}+01$ \\
$D=100$ & $\square$ & $1.078 \mathrm{E}+03$ & $9.148 \mathrm{E}+02$ & $1.010 \mathrm{E}+03$ & $4.789 \mathrm{E}+01$ \\
& $\triangle$ & $1.115 \mathrm{E}+03$ & $8.727 \mathrm{E}+02$ & $1.005 \mathrm{E}+03$ & $7.889 \mathrm{E}+01$ \\
\hline \multirow{2}{*}{$F_{5}$} & $\circ$ & $2.440 \mathrm{E}-01$ & $1.972 \mathrm{E}-02$ & $7.867 \mathrm{E}-02$ & $7.536 \mathrm{E}-02$ \\
$D=2$ & $\square$ & $3.254 \mathrm{E}-01$ & $2.932 \mathrm{E}-02$ & $1.156 \mathrm{E}-01$ & $1.026 \mathrm{E}-01$ \\
& $\triangle$ & $3.428 \mathrm{E}-01$ & $8.386 \mathrm{E}-03$ & $9.869 \mathrm{E}-02$ & $1.232 \mathrm{E}-01$ \\
\hline \multirow{2}{*}{$F_{5}$} & $\circ$ & $3.916 \mathrm{E}+02$ & $1.784 \mathrm{E}+02$ & $2.490 \mathrm{E}+02$ & $6.166 \mathrm{E}+01$ \\
$D=100$ & $\square$ & $4.518 \mathrm{E}+02$ & $1.879 \mathrm{E}+02$ & $3.076 \mathrm{E}+02$ & $7.058 \mathrm{E}+01$ \\
& $\triangle$ & $4.788 \mathrm{E}+02$ & $2.579 \mathrm{E}+02$ & $3.769 \mathrm{E}+02$ & $7.286 \mathrm{E}+01$ \\
\hline \multirow{2}{*}{$F_{6}$} & $\circ$ & $1.890 \mathrm{E}+01$ & $4.756 \mathrm{E}-07$ & $1.890 \mathrm{E}+00$ & $5.977 \mathrm{E}+00$ \\
$D=2$ & $\square$ & $1.993 \mathrm{E}+01$ & $2.050 \mathrm{E}-06$ & $3.965 \mathrm{E}+00$ & $8.359 \mathrm{E}+00$ \\
& $\triangle$ & $2.030 \mathrm{E}+01$ & $7.201 \mathrm{E}-06$ & $5.799 \mathrm{E}+00$ & $9.361 \mathrm{E}+00$ \\
\hline \multirow{2}{*}{$F_{6}$} & $\circ$ & $2.052 \mathrm{E}+01$ & $1.569 \mathrm{E}+01$ & $1.774 \mathrm{E}+01$ & $1.468 \mathrm{E}+00$ \\
$D=100$ & $\square$ & $2.021 \mathrm{E}+01$ & $1.898 \mathrm{E}+01$ & $1.949 \mathrm{E}+01$ & $4.244 \mathrm{E}-01$ \\
& $\triangle$ & $2.004 \mathrm{E}+01$ & $1.735 \mathrm{E}+01$ & $1.919 \mathrm{E}+01$ & $8.287 \mathrm{E}-01$ \\
\hline \multirow{2}{*}{$F_{7}$} & $\circ$ & $1.693 \mathrm{E}+03$ & $1.690 \mathrm{E}+03$ & $1.691 \mathrm{E}+03$ & $6.175 \mathrm{E}-01$ \\
$D=2$ & $\square$ & $1.694 \mathrm{E}+03$ & $1.690 \mathrm{E}+03$ & $1.691 \mathrm{E}+03$ & $1.057 \mathrm{E}+00$ \\
& $\triangle$ & $1.692 \mathrm{E}+03$ & $1.690 \mathrm{E}+03$ & $1.691 \mathrm{E}+03$ & $8.257 \mathrm{E}-01$ \\
\hline \multirow{2}{*}{$F_{7}$} & $\circ$ & $6.319 \mathrm{E}+02$ & $5.455 \mathrm{E}+02$ & $5.944 \mathrm{E}+02$ & $2.676 \mathrm{E}+01$ \\
$D=100$ & $\square$ & $7.220 \mathrm{E}+02$ & $5.453 \mathrm{E}+02$ & $6.383 \mathrm{E}+02$ & $4.941 \mathrm{E}+01$ \\
& $\triangle$ & $6.691 \mathrm{E}+02$ & $5.575 \mathrm{E}+02$ & $6.163 \mathrm{E}+02$ & $3.442 \mathrm{E}+01$ \\
\hline
\end{tabular}

possible time complexity of the algorithm. In order to have much more understandable and comparable results the tuning of behavioural parameters was also carried out with the use of expert's knowledge and trial and error procedure. In the first case, the suggestions proposed in [31] were applied (cognitive attraction $=0.5$, social attraction $=1.25$ ). In the second case values of behavioural parameters were changed several times for obtaining satisfactory solutions. The optimization process was run ten times for each case and afterwards the results were averaged. Overall, the comparison results of metaoptimization (०) and classic strategies $(\square, \triangle$ ) for adjusting behavioural parameter values were included in Tab. II. The most important statistic measures such as AVG and STD show that the best option is to find optimal values of behavioural parameters by means of the meta-optimization method. It is also confirmed by results presented in Figs. 2b,d,f-l (for functions $F_{3}, F_{6}$ and $F_{7}, D=2$ and $D=100$, respectively). These plots demonstrate mean values of the best scores of the cost function (MS) vs. the number of function evaluations (FES) for investigated cases. Each of these examples illustrates the effectiveness of the proposed meta-optimization method when it is compared to classic approaches.

\section{B. Description of the damage identification problem}

The testing data was achieved during experimental measurements (modal analysis) of artificially damaged square composite plates clamped on the edges. The damages with depth of $0.5 \mathrm{~mm}$ (ca. 19\% of total thickness) were included using numerical milling machine. In the first case there was through-the-length crack, in the second case there was a spatial square damage and in the last case there were multiple 

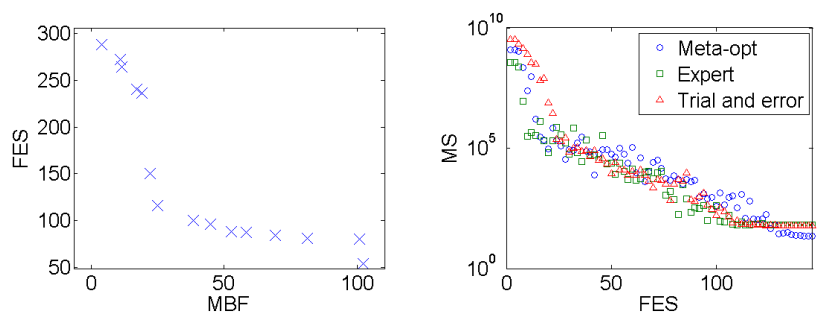

(a) Meta-opt. of $F_{3}(D=2)$

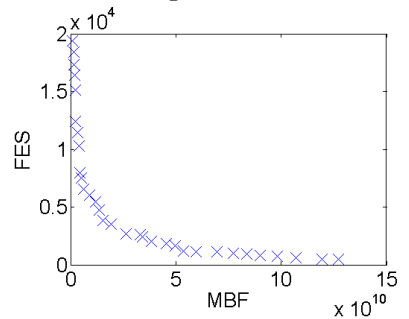

(c) Meta-opt. of $F_{3}(D=100)$

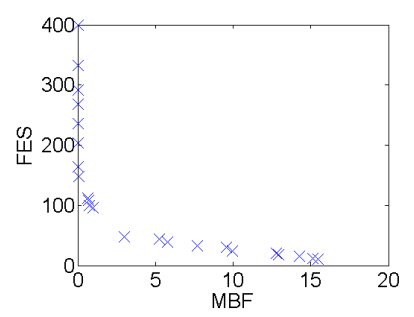

(e) Meta-opt. of $F_{6}(D=2)$

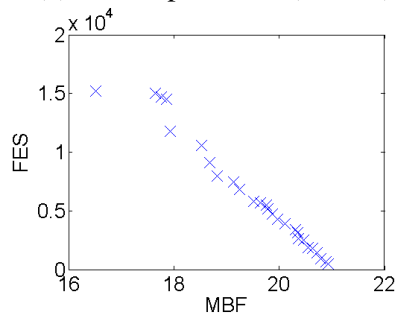

(g) Meta-opt. of $F_{6}(D=100)$

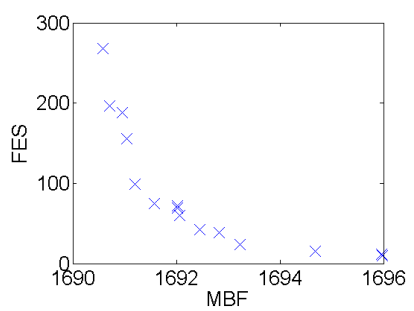

(i) Meta-opt. of $F_{7}(D=2)$

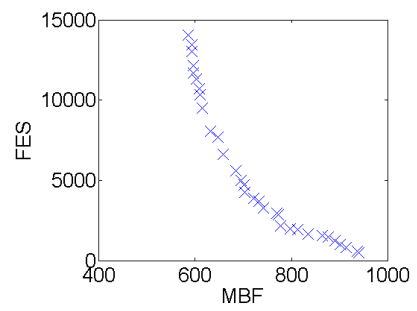

(k) Meta-opt. of $F_{7}(D=100)$ (b) Opt. of $F_{3}(D=2)$

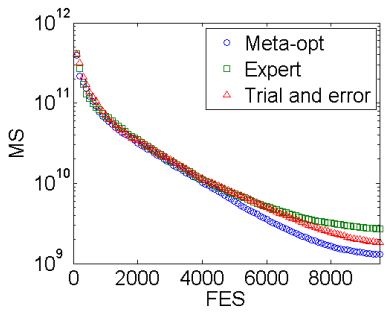

(d) Opt. of $F_{3}(D=100)$

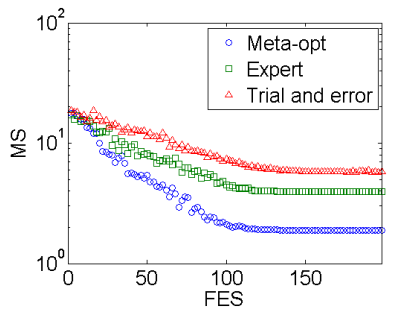

(f) Opt. of $F_{6}(D=2)$

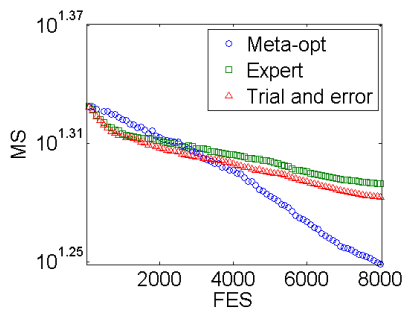

(h) Opt. of $F_{6}(D=100)$

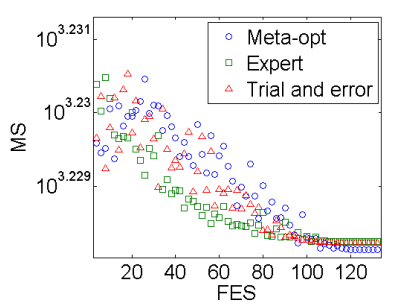

(j) Opt. of $F_{7}(D=2)$

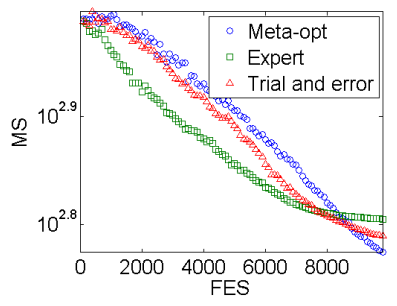

(1) Opt. of $F_{7}(D=100)$

Fig. 2: The comparison results of using meta-optimization, expert, trail and error procedures in selection of the behavioural parameter values
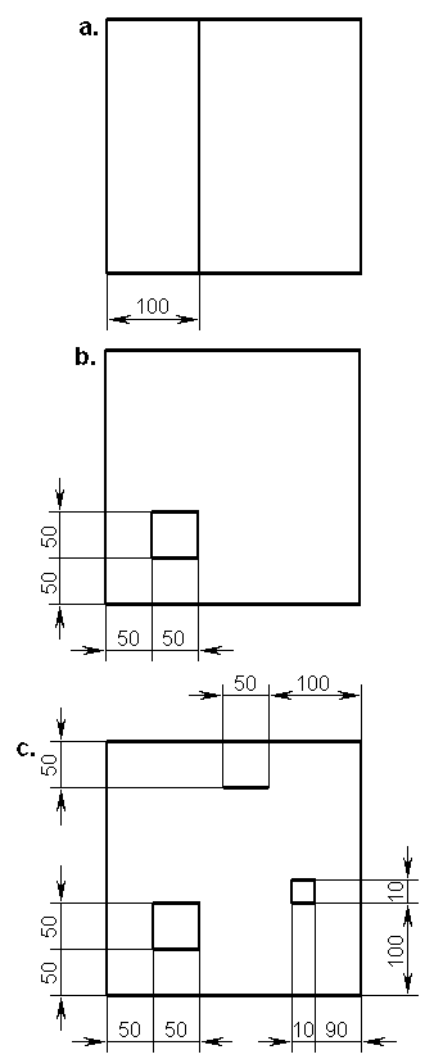

Fig. 3: Damaged plates considered in the analysis

damages: one small crack and two square spatial damages. The specific dimensions of damaged plates were presented in Fig. 3. During the scanning procedure the displacements in the net of $64 \times 64$ equidistant points were collected. The details of experimental setup and performing measurements can be found in [18].

First five modal shapes of each investigated case were considered in further analysis. Then, the collected data was exported to MATLAB ${ }^{\circledR}$ environment.

\section{Results of damage identification}

In this case study, the meta-optimization process was carried out on data collected using finite element (FE) analysis. The numerical models were prepared according to the geometry specification presented in Fig. 3 using MSC.Marc/Mentat ${ }^{\circledR}$ FE commercial software. The plates were modelled as 3D structures with the lay-up of a laminate and respective material properties presented in [17] and meshed using 8-node hexagonal elements. The boundary conditions were the same as for experimental study, i.e. all of the edges were clamped. The analyses were defined as normal mode evaluation, where the displacements in normal direction to the surface of a plate in $64 \times 64$ equidistant points of the first five modal shapes were considered for further studies. Due to the above-presented problem definition the numerical data was used as training data. 


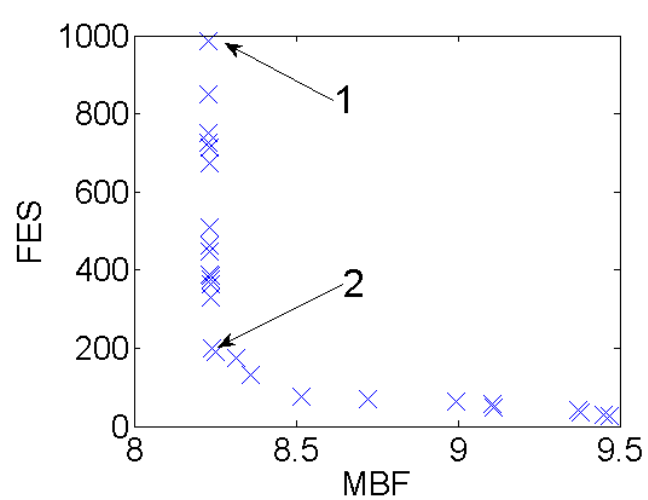

Fig. 4: Pareto front with optimal solutions obtained in the meta-optimization process

TABLE III: Optimal values of behavioural parameters for the damage identification purpose

\begin{tabular}{|c|c|c|c|c|c|c|}
\hline No. & $\begin{array}{c}\text { Cognitive } \\
\text { attraction }\end{array}$ & $\begin{array}{c}\text { Social } \\
\text { attraction }\end{array}$ & Generations & $\begin{array}{c}\text { Population } \\
\text { size }\end{array}$ & FES & MBF \\
\hline 1 & 0.4324 & 0.8390 & 34 & 29 & 986 & 8.2307 \\
\hline 2 & 0.1353 & 1.0078 & 25 & 8 & 200 & 8.2411 \\
\hline
\end{tabular}

The extreme was found with the help of the PSO-OAE. The cost function in the PSO-OAE was evaluated using the indirect utility function (9) for three damaged plates at the same time. The lower and upper boundaries for $\alpha$ and $\tau$ in the PSO-OAE were assumed taking into account wavelet's properties $\left(\alpha^{(L)}=\right.$ $\tau^{(L)}=0, \alpha^{(U)}=2.5, \tau^{(U)}=6$ ). Behavioural parameters were selected during the meta-optimization process realized by means of MOEA-MAC. The boundaries for these variables were declared in such a way, that the population size as well as the total number of generations were equal from 5 to 35 , the cognitive and social attraction were equal from 0 to 4 . The rest of the features of MOEA-MAC were selected in the same way as in the previous case.

The key results from the meta-optimization were shown in Fig. 4. This plot presents the Pareto front that means the set of non-dominated solutions. Due to the form of the plot it was possible that two optimal solutions were chosen for the further analysis. The values of behavioural parameters for these cases were included in Tab. III. It should be easily noted, that almost the same values of MBF can be achieved with the smaller number of function evaluations FES.

The damage identification experiments and the main optimization process were also repeated for real-world data. The values of behavioural parameters in this instance were the same as for the numerical data. Despite this, it was enough to obtain the high performance of damage identification for real measurements. Figs. 5a, 6a, 7a illustrate the main optimization processes (PSO-OAE) conducted applying the 1st and 2nd set of optimal values of behavioural parameters. In this way, it was possible for each case to obtain such values of parameters for which the total number of function evaluations was not larger than 200 to be enough to find the final solution.

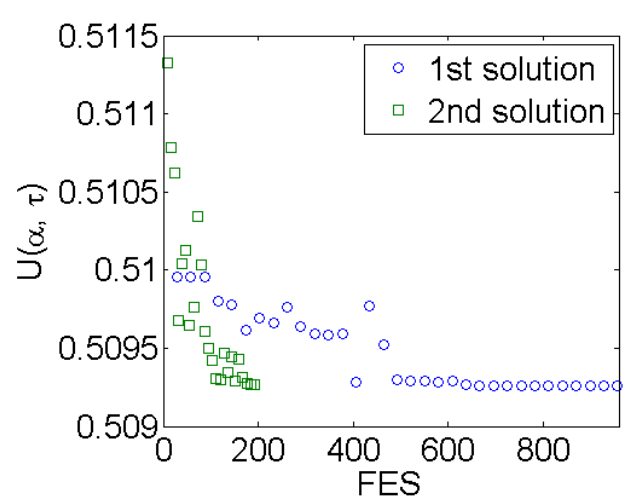

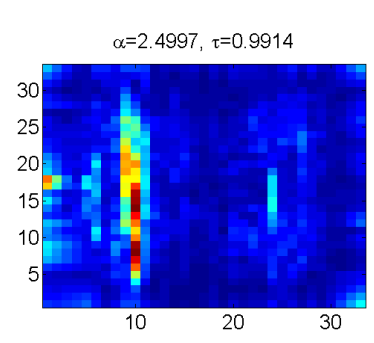

(b) for the 1 st solution (a)

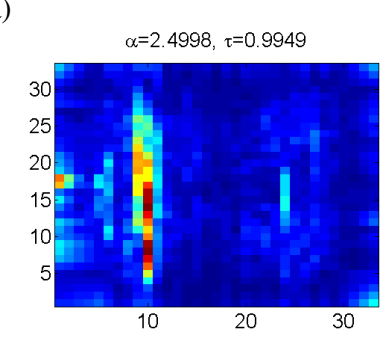

(c) for the 2nd solution
Fig. 5: The comparison of two non-dominated solutions from meta-optimization in the identification of the first damage

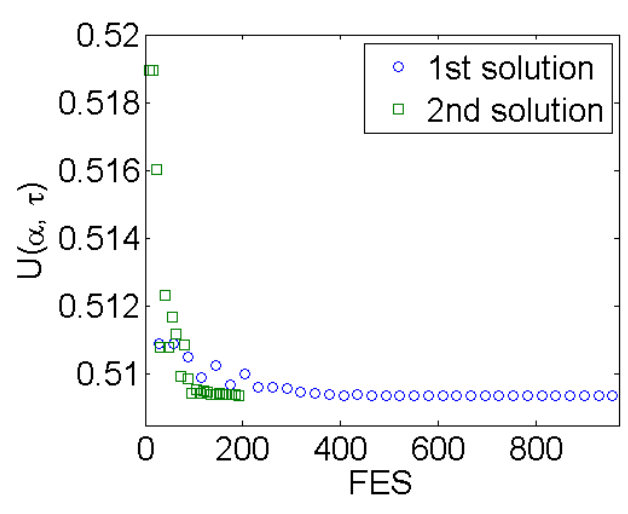

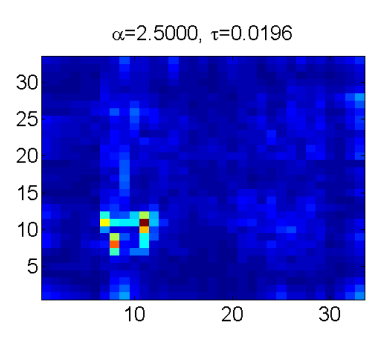

(b) for the 1st solution (a)

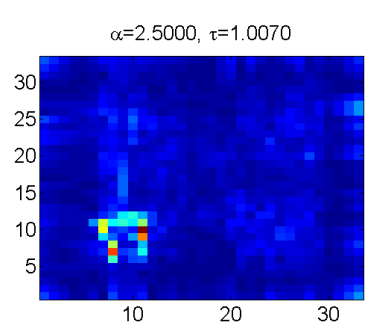

(c) for the 2nd solution
Fig. 6: The comparison of two non-dominated solutions from meta-optimization in the identification of the second damage 


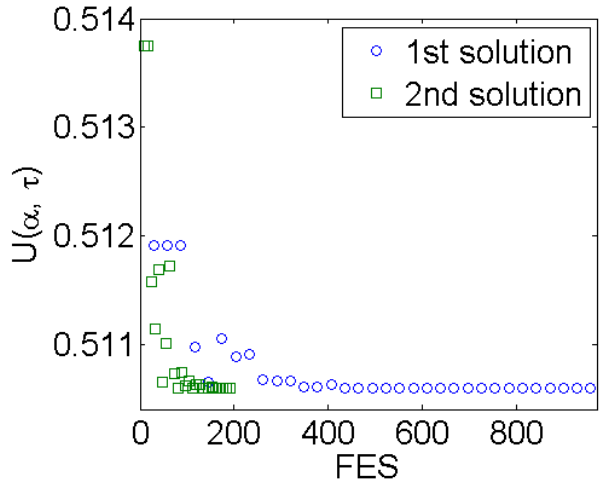

(a)

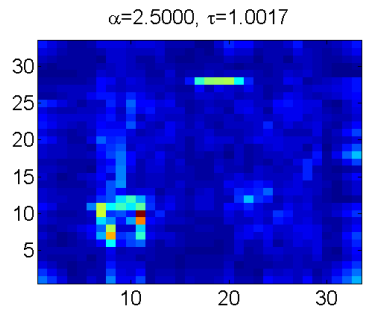

(b) for the 1 st solution

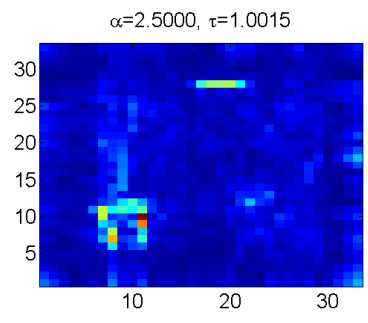

(c) for the 2nd solution
Fig. 7: The comparison of two non-dominated solutions from meta-optimization in the identification of the third damage

From the Pareto fronts presented in Figs. 5a-7a one can conclude that the compromise between time complexity of computations and the accuracy of the final solution has been reached. It is also conducted by the obtained results (see Figs. 5b,c-7b,c), where the optimized parameters of a wavelet in solutions obtained by the proposed method have almost identical values with those obtained using typical optimization procedure (1st solution), however these values were obtained much earlier in the 2 nd solution than in the 1 st one. It could be also noticed that the obtained values for $\alpha$ and $\tau$ cause that the applied wavelet has a great filtering ability and simultaneously short effective support, which cause that all of the damages were detected and located properly. The optimization algorithm solves the problem with selection of wavelet parameters, which influences much on the ability of damage detection and localization, i.e. in the case of underestimated value of $\alpha$ the obtained sets of coefficients are highly biased by the rests of a filtered signal due to inappropriate filtration, while in the case of overestimation of $\alpha$ the resulted sets of coefficients are blurred in the locations of singularities (damages) due to the power losses of a wavelet with the wide effective support, which makes the decision process about damage presence and location difficult and unambiguous.

\section{Conclusions}

In this paper, the authors proposed a new method for finding optimal values of behavioural parameters of the optimization procedure that is used in order to identify damages in composite structures. The proposed approach is based on the metaoptimization concept. The novelty of the proposed method depends on that the meta-optimization can be realized using multi-objective cost functions.

The preliminary verification of the elaborated approach in optimization tasks was carried out for well-known benchmark functions. The results achieved in this part of investigations demonstrate the capabilities of the approach for solving different kinds of optimization problems. The fundamental verification was conducted for the experimental data measured during tests on the artificially damaged composite plates. The problem of optimization of wavelet parameters applied for the structural damage assessment in composites was studied before by the authors, but the meta-optimization approach allows to obtain several new advantages. The parameters of optimization algorithm do not require to be known since they are determined by the meta-optimization procedure. This excludes the difficulties of determination of these parameters, which are often difficult to achieve and automates the method. Moreover, different cases of damages were analyzed together, which allows for the determination of global parameters of the applied fractional B-spline wavelets and guarantee the best possible results for the damage detection and localization problems. Finally, the compromise between the time complexity of computations and the accuracy of the final solution could be reached in the automated manner.

\section{REFERENCES}

[1] C.-C. Chang and L.W. Chen, "Damage Detection of a Rectangular Plate by Spatial Wavelet Based Approach," Appl. Acoust., vol. 65, 2004 pp. 819-832, http://dx.doi.org/10.1016/j.apacoust.2004.01.004.

[2] E. Douka, S. Loutridis and A. Trochidis, "Crack Identification in Plates Using Wavelet Analysis," J. Sound Vib., vol. 270, 2004, pp. 279-295, http://dx.doi.org/10.1016/S0022-460X(03)00536-4.

[3] Y. Huang, D. Meyer and S. Nemat-Nasser, "Damage Detection with Spatially Distributed 2D Continuous Wavelet Transform," Mech. Mater. vol. 41, 2009, pp. 1096-1107, http://dx.doi.org/10.1016/j.mechmat.2009. 05.006 .

[4] W. Fan and P. Qiao, "A 2D Continuous Wavelet Transform of Mode Shape Data for Damage Detection of Plate Structures," Int. J. Solids Struct., vol. 46, 2009, pp. 4379-4395, http://dx.doi.org/10.1016/j.ijsolstr. 2009.08.022

[5] S. Zhong and S.O. Oyadiji, "Crack Detection in Simply-Supported Beams Without Baseline Modal Parameters by Stationary Wavelet Transform," Mech. Syst. Signal Process., vol. 21, 2007, pp. 1853-1884, http://dx.doi.org/10.1016/j.ymssp.2006.07.007.

[6] A. Katunin, "Modal-Based Non-Destructive Damage Assessment in Composite Structures Using Wavelet Analysis: A Review," Int. J. Compos. Mater., vol. 3, 2013, pp. 1-9, http://dx.doi.org/10.5923/s.cmaterials. 201310.01

[7] A. Katunin and F. Holewik, "Crack Identification in Composite Elements with Non-Linear Geometry Using Spatial Wavelet Transform," Arch. Civ. Mech. Eng., vol. 13, 2013, pp. 287-296, http://dx.doi.org/10.1016/ j.acme.2013.02.003

[8] L.H. Yam, Y.J. Jan and J.S. Jiang, "Vibration-Based Damage Detection for Composite Structures Using Wavelet Transform and Neural Network Identification," Compos. Struct., vol. 60, 2003, pp. 403-412, http://dx. doi.org/10.1016/S0263-8223(03)00023-0.

[9] M. Rucka and K. Wilde, "Neuro-Wavelet Damage Detection Technique in Beam, Plate and Shell Structures with Experimental Validation," $J$. Theor. Appl. Mech., vol. 48, 2010, pp. 579-604. 
[10] H. Hein and J. Feklistova, "Computationally Efficient Delamination Detection in Composite Beams Using Haar Wavelets," Mech. Syst. Signal Process., vol. 25, 2011, pp. 2257-2270, http://dx.doi.org/10.1016/ j.ymssp.2011.02.003.

[11] M. Krawczuk, A. Żak and W. Ostachowicz, "Genetic Algorithms in Fatigue Crack Detection,” J. Theor. Appl. Mech., vol. 39, 2001, pp. 815823.

[12] J. Xiang and M. Liang, "A Two-Step Approach to Multi-Damage Detection for Plate Structures," Eng. Fract. Mech., vol. 91, 2012, pp. 7386, http://dx.doi.org/10.1016/j.engfracmech.2012.04.028.

[13] J. Dumont, A. Hernández and G. Carrault, "Improving ECG Beats Delineation with an Evolutionary Optimization Process," IEEE Trans. Bio-Med. Eng., vol. 57, 2010, pp. 607-615, http://dx.doi.org/10.1109/ TBME.2008.2002157.

[14] A. Katunin, "Crack Identification in Composite Beam Using Causal Bspline Wavelets of Fractional Order," Model. Eng., vol. 15, 2013, pp. 57 63.

[15] M. Unser and T. Blu, "Fractional Splines and Wavelets," SIAM Rev., vol. 42, 2000, pp. 43-67, http://dx.doi.org/10.1137/S0036144598349435.

[16] K.N. Chaudhury and M. Unser, "Construction of Hilbert Transform Pairs of Wavelet Bases and Gabor-Like Transforms," IEEE Trans. Signal Process., vol. 57, 2009, pp. 3411-3425, http://dx.doi.org/10.1109/TSP. 2009.2020767.

[17] A. Katunin, "Damage Identification in Composite Plates Using TwoDimensional B-spline Wavelets," Mech. Syst. Signal Process., vol. 25, 2011, pp. 3153-3167, http://dx.doi.org/10.1016/j.ymssp.2011.05.015.

[18] A. Katunin and P. Przystałka, "Damage Assessment in Composite Plates Using Fractional Wavelet Transform of Modal Shapes with Optimized Selection of Spatial Wavelets," Eng. Appl. Artif. Intell., vol. 30, 2014, pp. 73-85, http://dx.doi.org/10.1016/j.engappai.2014.01.003.

[19] R.E. Mercer and J.R. Sampson, "Adaptive Search Using a Reproductive Meta-Plan,” Int. J. Syst. Cybern., vol. 7, 1977, pp. 215-228, http://dx. doi.org/10.1108/eb005486.

[20] J.J. Grefenstette, "Optimization of Control Parameters for Genetic Algorithms," IEEE Trans. Syst. Man Cybern., vol. 16, 1986, pp. 122128 , pp. 215-228, http://dx.doi.org/10.1109/TSMC.1986.289288.

[21] A.J. Keane, "Genetic Algorithm Optimization in Multi-Peak Prob- lems: Studies in Convergence and Robustness," Artificial Intelligence in Engineering, vol. 9, 1995, pp. 75-83, http://dx.doi.org/10.1016/ 0954-1810(95)95751-Q.

[22] T. Back, "Parallel Optimization of Evolutionary Algorithms," Proc. Int. Conf. on Evolutionary Computation, " 1994, pp. 418-427, http://dx.doi. org/10.1007/3-540-58484-6_285.

[23] S.K. Smit and A.E. Eiben, "Comparing Parameter Tuning Methods for Evolutionary Algorithms," Proc. IEEE Congress on Evolutionary Computation (CEC), 2009, pp. 399-406, http://dx.doi.org/10.1109/CEC. 2009.4982974.

[24] M. Meissner, M. Schmuker and G. Schneider, "Optimized Particle Swarm Optimization (OPSO) and Its Application to Artificial Neural Network Training," BMC Bioinformatics, vol. 7, 2006, 125, http://dx. doi.org/10.1186/1471-2105-7-125.

[25] J. Branke and J.A. Elomari, "Meta-Optimization for Parameter Tuning with a Flexible Computing Budget," Proc. 14th Annual Conf. on Genetic and Evolutionary Computation (GECCO12), Terence Soule (Ed.), New York, USA, 2012, pp. 1245-1252, http://dx.doi.org/10.1145/2330163. 2330336.

[26] K. Miya, M. Uesaka and Y. Yoshida, "Applied Electromagnetics Research and Application,” Prog. Nucl. Energ., vol. 32, 1998, pp. 179-194, http://dx.doi.org/10.1016/S0149-1970(97)00015-2.

[27] R.T. Marler and J.S. Arora, "Survey of Multi-Objective Optimization Methods for Engineering," Struct. Multidiscip. O., vol. 26, 2004 pp. 369-395, http://dx.doi.org/10.1007/s00158-003-0368-6.

[28] K. Deb, "Multi-Objective Optimization Using Evolutionary Algorithms," Wiley, 2009.

[29] S.M. Mikki and A.A. Kishk, "Particle Swarm Optimization: A PhysicsBased Approach," Morgan and Claypool, 2008.

[30] K. Tang, X. Yao, P. N. Suganthan, C. MacNish, Y. P. Chen, C. M. Chen, and Z. Yang, "Benchmark Functions for the CEC'2008 Special Session and Competition on Large Scale Global Optimization," Technical Report, Nature Inspired Computation and Applications Laboratory, USTC China, http://nical.ustc.edu.cn/cec08ss.php, 2007.

[31] M. Clerc and J. Kennedy, "The Particle Swarm - Explosion, Stability, and Convergence in a Multidimensional Complex Space," IEEE T. Evolut Comput., vol. 6, 2002, pp. 58-73, http://dx.doi.org/10.1109/4235.985692. 